\title{
CHERN CLASSES OF THE MODULI STACK OF CURVES
}

\author{
Gilberto Bini
}

\begin{abstract}
Here we calculate the Chern classes of $\overline{\mathcal{M}}_{g, n}$, the moduli stack of stable $n$-pointed genus $g$ curves. In particular, we prove that such classes lie in the tautological ring.
\end{abstract}

\section{Introduction}

Let $g$ and $n$ be non-negative integers such that $n>2-2 g$. We denote by $\overline{\mathcal{M}}_{g, n}$ the Deligne-Mumford stack of stable genus $g$ curves with $n$ marked points. More generally, if $P$ is a set with $n$ elements, it will be technically convenient to work with $\overline{\mathcal{M}}_{g, P}$, i.e., the stack of genus $g$ stable curves whose marked points are labelled by $P$. The natural projection $\varphi$ from the stack $\overline{\mathcal{M}}_{g, P}$ to the coarse moduli space $\bar{M}_{g, P}$ induces an isomorphism $\varphi_{*}$ at the level of Chow rings hereafter we shall only deal with rational coefficients. Following [6], we denote by $R^{*}\left(\bar{M}_{g, P}\right)$ the tautological ring of $\bar{M}_{g, P}$. By abuse of notation, we shall denote the image of $R^{*}\left(\bar{M}_{g, P}\right)$ under $\left(\varphi_{*}\right)^{-1}$ by the same symbol.

Tautological classes have been intensely studied in the last few years: see [18] for a synoptic survey on the most recent developments in this area. In particular, it is not at all clear which classes lie in the tautological ring. In fact, constructions of tautological or non-tautological classes can be very diverse in nature: combinatorial - as conjectured by Kontsevich and proved in, e.g., [12] - or purely algebro-geometric (e.g., [9]). As shown by Mumford in [14], the Grothendieck-Riemman-Roch Theorem allows one to express Chow classes in terms of tautological ones. Incidentally, this is done for the canonical class of $\overline{\mathcal{M}}_{g, P}$ in [13]. That calculation can be rephrased in terms of stacks. For foundational material on stacks we refer the reader to [19] and, especially for Chern classes, to [11].

In the present paper, we extend Mumford's work and calculate all Chern classes of the (smooth) moduli stack of curves, i.e., of the tangent bundle $\mathcal{T}_{\overline{\mathcal{M}}_{g, P}}$ to $\overline{\mathcal{M}}_{g, P}$. In spite of their geometric significance, these classes have not been hitherto computed. As above, we apply the Grothendieck-Riemann-Roch Theorem. In fact, we refine combinatorial arguments, and manage to get explicit formulae. This shows in particular that such classes are tautological, thus yielding new elements in $R^{*}\left(\bar{M}_{g, P}\right)$.

Received by the editors September 16, 2004. Revised April 14, 2005. 
Our presentation is rather concise since most of the theoretic material has been explored by several authors. We briefly recall the needed preliminaries in Section 2 and prove the main result in Section 3. Finally, we give some examples and compare our formulae with previous results.

Throughout, we shall work over the field of complex numbers.

\section{Preliminaries}

Let $\overline{\mathcal{M}}_{g, P}$ be the moduli stack of $P$-pointed stable curves of genus $g$. As usual, $\pi: \mathcal{C} \rightarrow \overline{\mathcal{M}}_{g, P}$ will denote the universal curve with sections $\sigma_{p}$ for $p \in P$. Set, further, $D:=\sum_{p \in P} \sigma_{p_{*}}(1)$. The universal cotangent classes on $\overline{\mathcal{M}}_{g, P}$ are defined as $\psi_{p}=c_{1}\left(\sigma_{p}^{*}\left(\omega_{\pi}\right)\right)$ for $p \in P$, where $\omega_{\pi}$ is the relative dualizing sheaf of $\pi$. The collection of all moduli stacks $\overline{\mathcal{M}}_{g, P}$ is equipped with some natural morphisms, namely:

$$
\xi_{G}: \prod_{v \in V} \overline{\mathcal{M}}_{g(v), l(v)} \rightarrow \overline{\mathcal{M}}_{g, P}
$$

where $G$ is a stable graph - see, e.g., [3]. For example, it is well known that the boundary $\partial \overline{\mathcal{M}}_{g, P}$ can be described in terms of the following morphisms:

$$
\begin{gathered}
\xi_{\text {irr }}: \overline{\mathcal{M}}_{g-1, P \cup\left\{q_{1}, q_{2}\right\}} \rightarrow \overline{\mathcal{M}}_{g, P}, \\
\xi_{h, A}: \overline{\mathcal{M}}_{h, A \cup\left\{r_{1}\right\}} \times \overline{\mathcal{M}}_{g-h, A^{c} \cup\left\{r_{2}\right\}} \rightarrow \overline{\mathcal{M}}_{g, P},
\end{gathered}
$$

where $0 \leq h \leq g, A \subseteq P$, and both $2 h-1+|A|$ and $2(g-h)-1+\left|A^{c}\right|$ are positive. Finally, let $\delta$ be the boundary class defined as

$$
\delta=\frac{1}{2} \xi_{i r r *}(1)+\frac{1}{2} \sum_{h} \sum_{A \subseteq P} \xi_{h, A *}(1) .
$$

Let $K=c_{1}\left(\omega_{\pi}(D)\right)$. Following [2], the Mumford classes on $\overline{\mathcal{M}}_{g, P}$ are defined as $\kappa_{m}=\pi_{*}\left(K^{m+1}\right)$. For $P=\emptyset$ their analogue was first introduced by Mumford in [14]. Another generalization of Mumford's $\kappa_{m}$ 's to the case of $P$-pointed curves is given by the classes $\widetilde{\kappa}_{m}=\pi_{*}\left(c_{1}\left(\omega_{\pi}\right)^{m+1}\right)$. As proved in [2], the following relationship holds:

$$
\kappa_{m}=\widetilde{\kappa}_{m}+\sum_{p \in P} \psi_{p}^{m}
$$

The Hodge bundle $\mathbb{E}$ on $\overline{\mathcal{M}}_{g, P}$ is defined as $\pi_{*} \omega_{\pi}$. From [5] and [14] we have

$$
\begin{gathered}
\operatorname{ch}(\mathbb{E})=g+\frac{1}{2} \sum_{m \geq 1} \frac{B_{2 m}}{(2 m) !}\left\{\widetilde{\kappa}_{2 m-1}+\right. \\
\xi_{i r r *}\left(\psi_{q_{1}}^{2 m-2}-\psi_{q_{1}}^{2 m-3} \psi_{q_{2}}+\ldots+\psi_{q_{2}}^{2 m-2}\right)+ \\
\left.\sum_{h=0}^{g} \sum_{A \subseteq P} \xi_{G_{h, A} *}\left(\psi_{r_{1}}^{2 m-2} \otimes 1-\psi_{r_{1}}^{2 m-3} \otimes \psi_{r_{2}}+\ldots+1 \otimes \psi_{r_{2}}^{2 m-2}\right)\right\},
\end{gathered}
$$


where $B_{2 m}$ are the Bernoulli numbers ${ }^{1}$. Note in particular that

$$
\operatorname{ch}_{1}(\mathbb{E})=c_{1}(\mathbb{E}):=\lambda=\frac{1}{12}\left(\kappa_{1}-\sum_{p \in P} \psi_{p}\right)+\delta .
$$

As first shown by Mumford in [14], the Grothendieck-Riemann-Roch Theorem (the G-R-R Theorem for short) can be applied to the universal curve $\pi: \mathcal{C} \rightarrow$ $\overline{\mathcal{M}}_{g, P}$. Alternatively, one can use the G-R-R Theorem stated in [17]. For the sake of completeness, we report the statement below.

Theorem 1. (G-R-R Theorem) Let $\mathcal{E}$ be a locally free sheaf on $\mathcal{C}$. Then

$$
\operatorname{ch}\left(\pi_{!} \mathcal{E}\right)=\pi_{*}\left(\operatorname{ch}(\mathcal{E}) T d^{\vee}\left(\Omega_{\pi}\right)\right),
$$

where $\Omega_{\pi}$ is the sheaf of relative Kähler differentials.

In Proposition $1, \operatorname{ch}(\mathcal{E})$ and $\operatorname{Td}(\mathcal{E})$ denote the Chern character and the dual Todd class of $\mathcal{E}$, respectively. Some formulas for these classes can be found, for instance, in [1]. Here, we just remark two basic facts. First, notice that

$$
\operatorname{ch}\left(\mathcal{E}^{*}\right)=r k(\mathcal{E})+\sum_{j \geq 1}(-1)^{j} \operatorname{ch}_{j}(\mathcal{E}) .
$$

Second, let $\mu=\left(1^{m_{1}} 2^{m_{2}} \ldots i^{m_{i}} \ldots\right)$ be a partition of weight $j$, where $j$ is a positive integer. Define $c h_{\mu}(\mathcal{E})$ to be the product $c h_{1}^{m_{1}}(\mathcal{E}) c h_{2}^{m_{2}}(\mathcal{E}) \ldots c h_{i}^{m_{i}}(\mathcal{E}) \ldots$ As proved in [10], (2.14'), the following holds:

$$
c_{j}(\mathcal{E})=\sum_{\mu \vdash j}(-1)^{j-l(\mu)} \prod_{r \geq 1} \frac{((r-1) !)^{m_{r}}}{m_{r} !} \operatorname{ch}_{\mu}(\mathcal{E}), \quad j \geq 1,
$$

where the sum ranges over all partitions $\mu$ of $j$, and $l(\mu)$ is the length of $\mu$.

Finally, we recall some properties of $Z$, the singular locus of $\pi$. For more details the reader is referred to [7]. $Z$ is a closed substack of codimension 2 in $\mathcal{C}$. Moreover, there exists a double ètale covering $\varepsilon: \widetilde{Z} \rightarrow Z$ obtained from the choice of the branches incident at the nodes corresponding to points in $Z$. Let $\iota: \widetilde{Z} \rightarrow \mathcal{C}$ be the natural composition. Denote by $\mathcal{L}$ and $\mathcal{L}^{\prime}$ the line bundles corresponding to the cotangent directions along the branches. Thus $\varepsilon^{*}\left(\mathcal{N}_{Z}\right)=\mathcal{L} \oplus \mathcal{L}^{\prime}$, where $\mathcal{N}_{Z}$ is the normal bundle of $Z$ in $\mathcal{C}$. Moreover, note that $\pi \circ \iota$ maps $\widetilde{Z}$ onto $\partial \overline{\mathcal{M}}_{g, P}$. In other words, we have

$$
\pi \circ \iota=\xi_{i r r}+\sum_{h} \sum_{A \subseteq P} \xi_{h, A}
$$

where both $2 h-1+|A|$ and $2(g-h)-1+\left|A^{c}\right|$ are positive.

\footnotetext{
${ }^{1}$ We recall that Bernoulli numbers are defined as follows:
}

$$
\frac{x}{e^{x}-1}=1-\frac{1}{2} x+\sum_{m \geq 1} \frac{B_{2 m}}{(2 m) !} x^{2 m}
$$




\section{The Chern character of $\overline{\mathcal{M}}_{g, P}$}

In this section we apply the G-R-R Theorem to the sheaf $\Omega_{\pi}(D) \otimes \omega_{\pi}$. By standard duality theorems and deformation theory [13], $\pi_{*}\left(\Omega_{\pi}(D) \otimes \omega_{\pi}\right)$ is the cotangent bundle on $\overline{\mathcal{M}}_{g, P}$. In the sequel, we closely follow [14], p. $302 \mathrm{ff}$.

We recall that

$$
\operatorname{ch}\left(\Omega_{\pi}(D)\right)=\operatorname{ch}\left(\omega_{\pi}(D)\right)-\operatorname{ch}\left(\mathcal{O}_{Z}\right)
$$

and

$$
T d^{\vee}\left(\Omega_{\pi}\right)=T d^{\vee}\left(\omega_{\pi}\right)\left(T d^{\vee}\left(\mathcal{O}_{Z}\right)\right)^{-1},
$$

where $\mathcal{O}_{Z}$ is viewed as a sheaf on $\mathcal{C}$. For the purpose of what follows, we need to determine the power series $\operatorname{ch}\left(\mathcal{O}_{Z}\right) T d^{\vee}\left(\mathcal{O}_{Z}\right)^{-1}$. Since $T d^{\vee}\left(\mathcal{O}_{Z}\right)^{-1}$ is a polynomial in the Chern characters $c_{k}\left(\mathcal{O}_{Z}\right)$, the G-R-R Theorem applied to $\iota$ yields a universal power series $\Theta$. For all $\nu: Y \rightarrow X$, an inclusion of a smooth codimension two subvariety in a smooth variety, the series $\Theta$ satisfies the following identity:

$$
\operatorname{ch}\left(\mathcal{O}_{Y}\right) T d^{\vee}\left(\mathcal{O}_{Y}\right)^{-1}=\nu_{*}\left(\Theta\left(c_{1}\left(\mathcal{N}_{Y}\right), c_{2}\left(\mathcal{N}_{Y}\right)\right)\right) .
$$

To compute $\Theta$, take $Y=D_{1} D_{2}$. Then the exact sequence

$$
0 \rightarrow \mathcal{O}_{X}\left(-D_{1}-D_{2}\right) \rightarrow \mathcal{O}_{X}\left(-D_{1}\right) \oplus \mathcal{O}_{X}\left(-D_{2}\right) \rightarrow \mathcal{O}_{X} \rightarrow \mathcal{O}_{Y} \rightarrow 0
$$

yields

$$
\operatorname{ch}\left(\mathcal{O}_{Y}\right)=\left(1-e^{-D_{1}}\right)\left(1-e^{-D_{2}}\right) .
$$

By [14], p. 303, we have

$$
T d^{\vee}\left(\mathcal{O}_{Y}\right)^{-1}=\frac{D_{1} D_{2}}{D_{1}+D_{2}} \frac{1-e^{-D_{1}-D_{2}}}{\left(1-e^{-D_{1}}\right)\left(1-e^{-D_{2}}\right)} .
$$

Therefore, we get

$$
\operatorname{ch}\left(\mathcal{O}_{Y}\right) T d^{\vee}\left(\mathcal{O}_{Y}\right)^{-1}=D_{1} D_{2} \sum_{j \geq 1}(-1)^{j-1} \frac{\left(D_{1}+D_{2}\right)^{j-1}}{j !} .
$$

Thus, the following holds:

$$
\Theta\left(D_{1}+D_{2}, D_{1} D_{2}\right)=\sum_{j \geq 1}(-1)^{j-1} \frac{\left(D_{1}+D_{2}\right)^{j-1}}{j !} .
$$

As noted in [7], (13) can be applied to the morphism $\iota: \widetilde{Z} \rightarrow \mathcal{C}$ as well. In this case, we need to replace the Chern classes of $\mathcal{N}_{Z}$ with those of $\varepsilon^{*} \mathcal{N}_{Z}$.

We now state the main result of this section. 
Theorem 2. The Chern character of the cotangent bundle on $\overline{\mathcal{M}}_{g, P}$ is given by $\left(17 \Varangle h\left(\mathcal{T}_{\mathcal{M}_{g, P}}\right)=\sum_{j \geq 1} \frac{\kappa_{j-1}}{j !}+\frac{1}{2} \sum_{t \geq 1} \frac{\kappa_{t}}{t !}-\sum_{m \geq 3} a_{m} \kappa_{m-1}+\right.$

$$
\operatorname{ch}(\mathbb{E})-1-\frac{1}{2} \xi_{i r r} *\left(\Xi^{(1)}\right)-\frac{1}{2} \sum_{h=0}^{g} \sum_{A \subseteq P} \xi_{G_{h, A}} *\left(\Xi^{(2)}\right),
$$

where

$$
\begin{gathered}
a_{m}=\sum_{h=1}^{\left\lfloor\frac{\left.\frac{m-1}{2}\right\rfloor}{2} \frac{B_{2 h}}{(2 h) !(m-2 h) !},\right.} \\
\Xi^{(1)}=\sum_{k \geq 1}(-1)^{k-1} \frac{\left(\psi_{q_{1}}+\psi_{q_{2}}\right)^{k-1}}{k !}
\end{gathered}
$$

and

$$
\Xi^{(2)}=\sum_{k \geq 1}(-1)^{k-1} \frac{\left(\psi_{r_{1}} \otimes 1+1 \otimes \psi_{r_{2}}\right)^{k-1}}{k !}
$$

Proof. By (10) and (11), we have

$$
\begin{aligned}
(21) \operatorname{ch}\left(\left(\Omega_{\pi}(D) \otimes \omega_{\pi}\right) T d^{\vee}\left(\Omega_{\pi}\right)\right) & =\operatorname{ch}\left(\omega_{\pi}\right) T d^{\vee}\left(\omega_{\pi}\right) T d^{\vee}\left(\mathcal{O}_{Z}\right)^{-1} \operatorname{ch}\left(\omega_{\pi}(D)\right) \\
& -\operatorname{ch}\left(\omega_{\pi}\right) T d^{\vee}\left(\omega_{\pi}\right) T d^{\vee}\left(\mathcal{O}_{Z}\right)^{-1} \operatorname{ch}\left(\mathcal{O}_{Z}\right) .
\end{aligned}
$$

As proved in [2], the first Chern class of $\omega_{\pi}(D)$ is equal to $\psi_{q}$. Here $\psi_{q}$ denotes the universal cotangent class on $\overline{\mathcal{M}}_{g, P \cup\{q\}}$ corresponding to the marked point $q$. Since $\mathcal{O}_{Z}$ is supported on $Z$ and the marked points are non-singular, we get

$$
\begin{aligned}
(22) \operatorname{ch}\left(\omega_{\pi}\right) T d^{\vee}\left(\omega_{\pi}\right) T d^{\vee}\left(\mathcal{O}_{Z}\right)^{-1} \operatorname{ch}\left(\omega_{\pi}(D)\right) & =\operatorname{ch}\left(\omega_{\pi}\right) T d^{\vee}\left(\omega_{\pi}\right) \operatorname{ch}\left(\omega_{\pi}(D)\right) \\
& +\operatorname{Td}^{\vee}\left(\mathcal{O}_{Z}\right)^{-1}-1,
\end{aligned}
$$

and

$$
\operatorname{ch}\left(\omega_{\pi}\right) T d^{\vee}\left(\omega_{\pi}\right) T d^{\vee}\left(\mathcal{O}_{Z}\right)^{-1} \operatorname{ch}\left(\mathcal{O}_{Z}\right)=T d^{\vee}\left(\mathcal{O}_{Z}\right)^{-1} \operatorname{ch}\left(\mathcal{O}_{Z}\right) .
$$

In particular, it is easy to check that (22) is equal to

$$
\operatorname{ch}\left(\omega_{\pi}\right) T d^{\vee}\left(\omega_{\pi}\right)\left[\operatorname{ch}\left(\omega_{\pi}(D)\right)-1\right]+\operatorname{ch}\left(\omega_{\pi}\right) T d^{\vee}\left(\Omega_{\pi}\right) .
$$

By definition, we have

$$
\begin{gathered}
\operatorname{ch}\left(\omega_{\pi}(D)\right)=e^{\psi_{q}} \\
\operatorname{ch}\left(\omega_{\pi}\right) T d^{\vee}\left(\omega_{\pi}\right)=e^{\left(\psi_{q}-D\right)} \frac{\psi_{q}-D}{e^{\left(\psi_{q}-D\right)}-1}=\frac{D-\psi_{q}}{e^{D-\psi_{q}}-1} .
\end{gathered}
$$


We recall that the formal expansion of the second power series in (25) is given by

$$
1+\frac{1}{2}\left(\psi_{q}-D\right)+\sum_{j \geq 1} \frac{B_{2 j}}{(2 j) !}\left(-\psi_{q}+D\right)^{2 j},
$$

where $B_{2 j}$ is the $(2 j)$-th Bernoulli number. Since $\psi_{q} \cdot D=0$, the first term of (24) is given by

$$
\sum_{j \geq 1} \frac{\psi_{q}^{j}}{j !}+\frac{1}{2} \sum_{t \geq 1} \frac{\psi_{q}^{t+1}}{t !}-\sum_{m \geq 3} a_{m} \psi_{q}^{m},
$$

where $a_{m}$ is defined in (18).

Let us apply $\pi_{*}$ to (21). The contribution in (24) yields

$$
\sum_{j \geq 1} \frac{\kappa_{j-1}}{j !}+\frac{1}{2} \sum_{t \geq 1} \frac{\kappa_{t}}{t !}-\sum_{m \geq 3} a_{m} \kappa_{m-1}+\operatorname{ch}(\mathbb{E})-1 .
$$

On the other hand, the contribution in (23) is given by

$$
\frac{1}{2}(\pi \circ \iota)_{*}\left(\Theta\left(c_{1}\left(\varepsilon^{*} \mathcal{N}_{Z}\right), c_{2}\left(\varepsilon^{*} \mathcal{N}_{Z}\right)\right)\right) .
$$

By (9), this is equivalent to

$$
\frac{1}{2} \xi_{i r r *}\left(\Xi^{(1)}\right)+\frac{1}{2} \sum_{h=0}^{g} \sum_{A \subseteq P} \xi_{G_{h, A} *}\left(\Xi^{(2)}\right),
$$

where $\Xi^{(1)}$ and $\Xi^{(2)}$ are defined in (19) and (20), respectively.

Remark 3. By Theorem 2 and (6), we get

$$
\begin{gathered}
\operatorname{ch}_{0}\left(\mathcal{T}_{\overline{\mathcal{M}}_{g, P}}^{*}\right)=\operatorname{rk}\left(\mathcal{T}_{\mathcal{M}_{g, P}}^{*}\right)=3 g-3+n, \\
\operatorname{ch}_{1}\left(\mathcal{T}_{\overline{\mathcal{M}}_{g, P}}^{*}\right)=K{\overline{\mathcal{M}_{g, P}}}=13 \lambda+\sum_{p \in P} \psi_{p}-2 \delta,
\end{gathered}
$$

where $K_{\overline{\mathcal{M}}_{g, P}}$ is the canonical class of the stack $\overline{\mathcal{M}}_{g, P}$ and $\delta$ is defined in (4). These formulas agree with previous known results: see, e.g., [4], [13].

Remark 4. Note that Theorem 2 and Formula (7) give the Chern character of $\mathcal{T}_{\overline{\mathcal{M}}_{g, P}}$.

By Formula (8), we obtain an expression for the Chern classes of $\overline{\mathcal{M}}_{g, P}$.

Corollary 5. For $j \geq 1$, we have

$$
c_{j}\left(\overline{\mathcal{M}}_{g, P}\right)=\sum_{\mu \vdash j}(-1)^{j-l(\mu)} \prod_{r \geq 1} \frac{((r-1) !)^{m_{r}}}{m_{r} !} \operatorname{ch} h_{\mu}\left(\overline{\mathcal{M}}_{g, P}\right) .
$$


Example. We give some examples for low $j$ 's. In higher degrees, one can use John Stembridge's symmetric function package SF for maple [16]. For the sake of simplicity, we denote $\sum_{p} \psi_{p}$ by $\psi$. As noted in Remark 3, we have

$$
c_{1}\left(\overline{\mathcal{M}}_{g, P}\right)=-13 \lambda-\psi+2 \delta .
$$

¿From Corollary 5, we get

$$
\operatorname{ch}_{2}\left(\overline{\mathcal{M}}_{g, P}\right)=\frac{\kappa_{2}}{3}+\frac{1}{4} \xi_{\text {irr } *}\left(\psi_{q_{1}}+\psi_{q_{2}}\right)+\frac{1}{4} \sum_{h, A} \xi_{h, A}\left(\psi_{r_{1}} \otimes 1+1 \otimes \psi_{r_{2}}\right) .
$$

hence

$$
\begin{aligned}
c_{2}\left(\overline{\mathcal{M}}_{g, P}\right)= & \frac{1}{2}(-13 \lambda-\psi+2 \delta)^{2}-\frac{1}{3} \kappa_{2}-\frac{1}{4} \xi_{i r r *}\left(\psi_{q_{1}}+\psi_{q_{2}}\right)- \\
& \frac{1}{4} \sum_{h, A} \xi_{h, A *}\left(\psi_{r_{1}} \otimes 1+1 \otimes \psi_{r_{2}}\right) .
\end{aligned}
$$

Finally, the degree 3 Chern character is equal to

$$
\begin{aligned}
\operatorname{ch}_{3}\left(\overline{\mathcal{M}}_{g, P}\right)= & -\frac{\kappa_{3}}{12}-\operatorname{ch}_{3}(\mathbb{E})+\frac{1}{12} \xi_{i r r *}\left(\psi_{q_{1}}^{2}+\psi_{q_{1}} \psi_{q_{2}}+\psi_{q_{2}}^{2}\right)+ \\
& \frac{1}{12} \sum_{h, A} \xi_{h, A *}\left(\psi_{r_{1}}^{2} \otimes 1+\left(\psi_{r_{1}} \otimes \psi_{r_{2}}\right)+1 \otimes \psi_{r_{2}}^{2}\right) .
\end{aligned}
$$

Thus, we get

$$
\begin{aligned}
c_{3}\left(\overline{\mathcal{M}}_{g, P}\right)=[ & +13 \lambda+\psi-2 \delta]\left[\frac{1}{3} \kappa_{2}+\frac{1}{4} \xi_{i r r} *\left(\psi_{q_{1}}+\psi_{q_{2}}\right)\right. \\
& \left.+\frac{1}{4} \sum_{h, A} \xi_{h, A *}\left(\psi_{r_{1}} \otimes 1+1 \otimes \psi_{r_{2}}\right)\right]- \\
& \frac{1}{6}[-13 \lambda-\psi+2 \delta]^{3}+2 \operatorname{ch}_{3}\left(\overline{\mathcal{M}}_{g, P}\right) .
\end{aligned}
$$

As a result of Theorem 2 and the definition of tautological classes, we get new elements in the tautological ring of $\overline{\mathcal{M}}_{g, P}$. Precisely, the following holds.

Corollary 6. The Chern classes of $\overline{\mathcal{M}}_{g, P}$ are tautological.

\section{Acknowledgements}

I wish to express my gratitude to Enrico Arbarello for introducing me to an enumerative geometry of moduli of curves along the lines of seminal work by David Mumford [14]. I would also like to thank the referee for helpful remarks. 


\section{References}

[1] E. Arbarello, M.D.T. Cornalba, P. Griffiths, J. Harris,, Geometry of algebraic curves, I, Grundleheren der math. Wissenschaften, vol. 267, Springer-Verlag, New York, 1985.

[2] _ Combinatorial and algebro-geometric cohomology classes on the moduli spaces of curves, J. Algebraic Geom., 5 (1996) 705-749.

[3] Calculating cohomology groups of moduli spaces of curves via algebraic geometry, Inst. Hautes Études Sci. Publ. Math., 88 (1998) 97-127.

[4] G.Bini, C. Fontanari, Moduli of curves and spin structures via algebraic geometry. To appear in Trans. Amer. Math. Soc. (2004).

[5] G. Bini, Generalized Hodge classes on moduli spaces of curves, Beiträge Algebra Geom., 44 (2003), no. 2, 559-565.

[6] C. Faber, R. Pandharipande, Logarithmic series and Hodge integrals in the tautological ring, With an appendix by Don Zagier, Michigan Math. J., 48 (2000) 215-252.

[7] Hodge integrals and Gromov-Witten theory, Invent. Math., 139 (2000) 173-199.

[8] W. Fulton, Intersection Theory, 2nd edition, Springer-Verlag, New York, 1998.

[9] T. Graber, R. Pandharipande, Constructions of nontautological classes on moduli spaces of curves, Michigan Math. J., 51 (2003) 93-109.

[10] I.G. Macdonald, Symmetric functions and Hall polynomials, 2nd edition, Clarendon Press, Oxford, 1995.

[11] Yu.I. Manin, Frobenius manifolds, quantum cohomology, and moduli spaces, AMS Colloquium Publ. 47, Providence, RI, 1999.

[12] G. Mondello, Combinatorial classes on $\overline{\mathcal{M}}_{g, n}$ are tautological, Int. Math. Res. Not., 44 (2004) 2329-2390.

[13] J. Harris, D. Mumford, On the Kodaira dimension of the moduli space of curves. With an Appendix by William Fulton, Invent. Math., 67 (1982) 23-88.

[14] D. Mumford, Towards an enumerative geometry of the moduli space of curves, in Arithmetic and Geometry (M. Artin and J. Tate, eds.) Part II, Birkhäuser, 1983, pp. 271-328.

[15] _ Stability of projective varieties, Lectures given at the "Institut des Hautes tudes Scientifiques", Bures-sur-Yvette, March-April 1976. Monographie de l'Enseignement Mathmatique, No. 24. L'Enseignement Mathmatique, Geneva, 1977. pp 74.

[16] J. Stembridge, A Maple package for symmetric functions, http://www.math.lsa.umich.edu/ $\sim$ jrs/maple.html.

[17] B. Toen, Théorèmes de Riemann-Roch pour les champs de Deligne-Mumford, K-theory 18 (1999), 33-76.

[18] R. Vakil, The moduli space of curves and its tautological ring, Notices Amer. Math. Soc., 50 (2003), no. 6, 647-658.

[19] A. Vistoli, Intersection theory on algebraic stacks and on their moduli spaces, Invent. Math., 97 (1989), no. 3, 613-670.

Dipartimento di Matematica, Università degli Studi di Milano, Via C. Saldini 50, 20133 Milano, Italy.

E-mail address: gilberto.bini@mat.unimi.it 